\title{
INTEGRAÇÃO DO FRAMEWORK DE GARRETT COM AS ABORDAGENS LEAN UX E ÁGIL: EXEMPLO APLICADO AO DESENVOLVIMENTO DE UM APLICATIVO.
}

\section{GARRETT FRAMEWORK INTEGRATION WITH LEAN UX AND AGILE APPROACHES: EXAMPLE APPLIED TO THE DEVELOPMENT OF AN APPLICATION.}

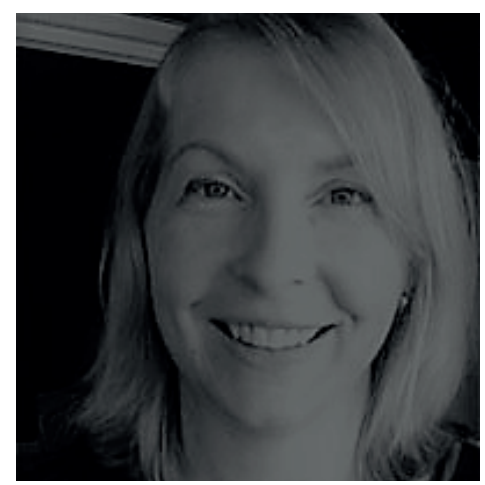

\section{Berenice Santos Gonçalves}

Doutora em Engenharia de Produção

Departamento de Expressão Gráfica, Universidade Federal de Santa Catarina

berenice@cce.ufsc.br

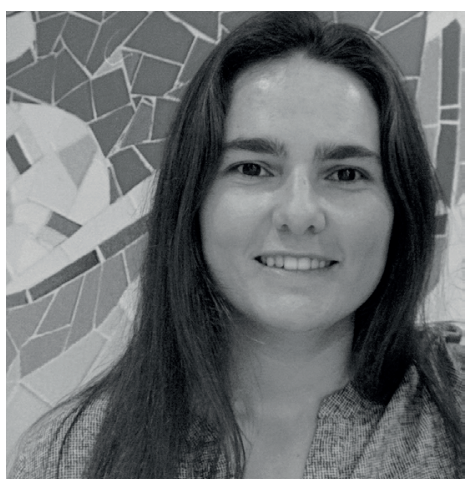

\author{
Andréa Bonette Ferrari \\ Bacharel em Design \\ Universidade Federal de Santa Catarina \\ andrea.bonette.ferrari@gmail.com
}




\section{RESUMO}

Este artigo apresenta a integração das abordagens Lean UX e Ágil com o framework de Garrett (2011), cuja motivação foi dar visibilidade às áreas do conhecimento importantes no design de sistemas interativos e, dessa forma, aumentar a confiança na prática profissional de designers de experiência do usuário. Os principais conceitos e valores das abordagens metodológicas Lean UX e Agile são apresentados, assim como a dinâmica do framework de Garrett. Ao final, apontam-se as contribuições trazidas pela aplicação da abordagem integrada no projeto da interface gráfica de um aplicativo Android, cujo objetivo é apoiar o acesso aos materiais bibliográficos de uma biblioteca universitária.

\section{PALAVRAS-CHAVE}

Lean UX; Ágil; Garrett.

\section{ABSTRACT}

This paper presents the integration of Lean UX and Agile approaches with Garrett's framework (2011), which motivation was to provide visibility to knowledge fields that are important to the design of interactive systems and thus enhance reliability during the professional practice of User Experience Designers. The main concepts and values of the Lean UX and Agile approaches are presented, as well as the dynamics of Garrett's framework. At the end, it is highlighted the contributions brought by the utilization of the integrated methodological approach in the user interface design of an Android application, which goal is to support the access to bibliographic material of a university library.

\section{KEYWORDS}

Lean UX; Agile; Garrett.

\section{INTRODUÇÃO}

Em um mercado de consumo cada vez mais competitivo, marcado pelo lançamento de tecnologias e produtos em ciclos cada vez mais curtos, precedidos ou sucedido por transformações de comportamento dos usuários, é necessário adotar processos orientados a diminuir riscos e incertezas ao longo de projetos de desenvolvimento de produtos. Especialmente nos projetos de sistemas interativos, riscos e incertezas vão desde entregar um produto que não atende as necessidades e expectativas dos usuários até a utilização de tecnologias de difícil manutenção. 
Entrevistar usuários, testar alternativas, protótipos e o próprio produto, entre outros métodos aplicados para obter e incorporar o feedback de usuários, não são suficientes por si só para diminuir riscos e incertezas. Muitos projetos falham porque realizam pesquisas com usuários apenas no início e/ou no final do projeto.

De acordo com a norma ISO 9241 - Parte 210 (2011, p. 9), "o projeto mais adequado para um sistema interativo normalmente não pode ser alcançado sem iteração", sem buscar regularmente o feedback de usuários e incorporá-los a especificações, requisitos, protótipos e produtos. Cabe destacar que "uma metodologia de design iterativa não envolve substituir cegamente elementos de interface por novas ideias alternativas de design." (NIELSEN, 1993, s.p.)

Metodologias ágeis (Agile), como o Scrum e o Kanban, ou influenciadas pela abordagem Ágil, como o Lean UX, são estruturadas em processos iterativos, nos quais são previstos vários momentos, métodos e técnicas para promover o contato com usuários (ASHMORE; RUNYAN, 2014; GOTHELF; SEIDEN, 2013). São metodologias contemporâneas, praticadas por todos os tipos e tamanhos de organizações, que entregam diferentes produtos e valores ao mercado consumidor. De acordo com relatório anual de 2015 preparado pela agência independente Analisys.Net Research e patrocinado pela VersionOne (2016, p. 6), 95\% das organizações ao redor do mundo, nas quais trabalham os quase 4 mil pesquisados, praticam metodologias ágeis.

As contribuições das diversas áreas do conhecimento (disciplinas) importantes no projeto de um sistema interativo - design de interação, design visual, arquitetura da informação e navegação, ergonomia e fatores humanos, dentre outras tantas -, no entanto, são diluídas em diversas técnicas, métodos e artefatos próprios de cada metodologia ágil e, portanto, ficam implícitas nos processos. Assim, especialmente designers de experiência do usuário podem sentir-se inseguros durante um projeto ágil quando refletem sobre se alguma dessas disciplinas foi negligenciada.

Neste trabalho, é apresentada a integração das abordagens metodológicas Lean UX (GOTHELF; SEIDEN, 2013) e Ágil com o framework conceitual de Garrett (2011) a fim de dar visibilidade às disciplinas importantes ao design de sistemas interativos e, assim, aumentar a confiança durante a prática profissional do designer de experiência do usuário. Esta integração foi aplicada no desenvolvimento da interface de um aplicativo móvel para apoiar o acesso aos materiais bibliográficos de um biblioteca universitária. 


\section{REVISÃO BIBLIOGRÁFICA}

\subsection{OS PLANOS DE GARRETT}

O framework criado por Garrett (2011) situa as diversas atividades relacionados com design de experiência do usuário (UX design) permeando o processo de desenvolvimento de um website, percebido tanto como uma interface de software - no qual usuários realizam tarefas para atingir seus objetivos - quanto como um sistema de hipertexto - no qual a informação é o foco de interesse do usuário e como este a compreende (GARRETT, 2011, p. 28). Este framework está estruturado em

Cinco planos [ou etapas] - estratégia, escopo, estrutura, esqueleto e superfície - [...] para abordar os problemas da experiência do usuário e as ferramentas utilizadas para resolvê-los. (GARRETT, 2011, p.23)

Em cada plano, cada uma das disciplinas importante no projeto de uma interface digital entre em ação; nenhuma delas é negligenciada, portanto. Esta é exatamente a principal contribuição de Garrett (2011): a construção de um panorama abrangente, porém de consistência mais teórica, de todas as disciplinas essenciais no projeto da experiência do usuário com um produto. A Figura 1 resume a contribuição de cada plano, aplicados sequencialmente de baixo para cima, de práticas projetuais que resultam em artefatos abstratos para aquelas que resultam em artefatos concretos.

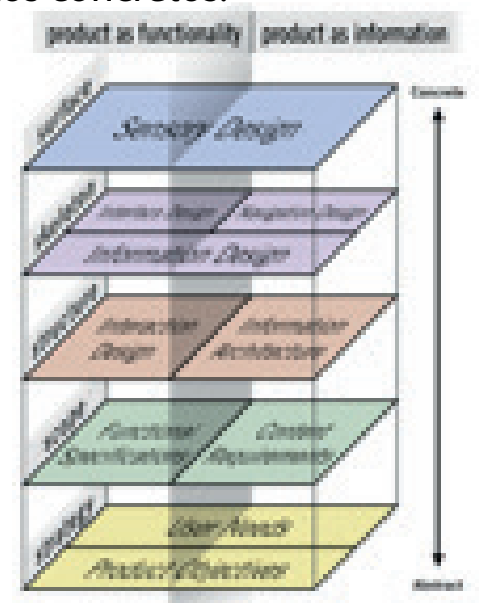

Figura 1: Os planos do framework de Garrett , 2011, p.29.

Fonte: Garrett, 2011, p. 29

Muito embora o framework conceitual de Garrett (2011) auxilie na compreensão e na organização das atividades de UX Design no processo de desenvolvimento de um website, sua estrutura engessada em etapas aparentemente 
lineares pode comprometer o sucesso do produto quando lançado no mercado cada vez mais dinâmico e exigente.

Garrett (2011, p. 22) alerta para o fato de que as decisões tomadas em um plano são influenciadas pelas decisões do plano anterior e limitam as decisões do plano posterior, mas não significa que estas decisões sejam intocáveis uma vez tomadas. Há espaço para reavaliá-las, especialmente decisões tomadas em planos consecutivos (2011, p. 24). No entanto, no mercado atual é necessário adotar processos focados mais explicitamente em iteração.

\section{2 ÁGIL E LEAN UX}

A proposta da abordagem Ágil surgiu como resposta à insatisfação de desenvolvedores com relação a processos lineares de desenvolvimento baseados em entregáveis e em documentação pesada (ASHMORE; RUNYAN, 2014, Seção "The Beginnings of Software Development as Methodology"). De acordo com Ashmore e Runyan (2014, Seção "The Beginnings of Software Development as Methodology"), "Ágil é um termo abrangente que inclui abordagens iterativas para desenvolvimento de software que adotam os valores do Manifesto para Desenvolvimento Ágil de Software".

O "Manifesto para Desenvolvimento Ágil de Software" (BECK et al., 2001) propõe quatro valores para guiar o desenvolvimento de software:

Indivíduos e interações mais que processos e ferramentas

Software em funcionamento mais que documentação abrangente

Colaboração com o cliente mais que negociação de contratos

Responder a mudanças mais que seguir um plano

Ou seja, mesmo havendo valor nos itens à direita, valorizamos mais os itens à esquerda. (BECK et al., 2001).

Dentre as várias metodologias ágeis - Extreme Programming (XP), Scrum, Feature-Driven Development, Lean Software Development, Kanban Method, dentre outras -, aquela mais praticada atualmente é o Scrum (VERSIONONE, 2016, p. 9). No Scrum, adota-se o formato de documentação de requisitos de projeto denominado user story, que ajuda a visualizar quem é o usuário, o que ele faz na interface e qual seu objetivo (ASHMORE; RUNYAN, 2014, Seção"Agile Requirements in Scrum"): Como <persona $>$, eu quero <algo $>$, para que <objetivo/ razão $>$.

O backlog (reserva) é a coleção priorizada de user stories e serve para compor e documentar objetivos e requisitos. Em um projeto ágil, há contato frequente com o público-alvo e, com isso, vai-se aprendendo com os usuários. Descobertas são comuns e ajudam a manter o produto na direção da satisfação de 
interesses e necessidades do usuário e objetivos e viabilidade do negócio. $\mathrm{O}$ aprendizado e as descobertas tornam o backlog um documento vivo, sendo continuamente atualizado e revisado: user stories são alteradas, repriorizadas, incluídas ou eliminadas.

O sprint é outra ferramenta importante da metodologia Scrum. É um ciclo de desenvolvimento com duração de tempo fixa, mas que deve ser suficiente para projetar, implementar e testar uma determinada quantidade de user stories, por uma equipe composta por um número tal de designers, desenvolvedores e outros perfis profissionais, os quais tem disponível uma gama limitada de recursos técnicos. Normalmente, um sprint dura entre duas a quatro semanas (ASHMORE; RUNYAN, 2014, Seção "Agile Requirements in Scrum").

Apesar das ferramentas do Scrum serem adequadas para projetos de sistemas interativos na realidade do mercado, ainda é um desafio a integração das atividades UX nas diferentes metodologias ágeis, incluindo o Scrum (GOTHELF; SEIDEN, 2013, p. 91 e 95). A abordagem Lean UX é uma das tentativas bem-sucedidas de integrar as atividades de UX design em metodologias ágeis e inclusive tem o Ágil, além de Design Thinking e o método Lean Startup, como pilares (GOTHELF; SEIDEN, 2013, p. 5-7).

As atividades são as mesmas praticadas isoladamente por designers fora das abordagens ágeis ou do Lean UX: entrevistas com usuários, observação direta de comportamentos, prototipação, testes de usabilidade, brainstorm, dentre outras. A diferença essencial é que

[a abordagem] Lean UX é a prática de trazer à luz a verdadeira natureza de um produto mais rápido, de um modo colaborativo e multidisciplinar que reduz a ênfase na documentação minuciosa enquanto aumenta o foco na construção de uma compreensão compartilhada da real experiência do produto sendo projetada. (GOTHELF; SEIDEN, 2013, p. 7).

Os artefatos construídos coletivamente no Lean UX não são um fim - entregáveis a serem passados para o próximo na linha de produção -, mas sim um meio para construir um entendimento coletivo sobre o que é ou o que será o produto. São desde post-its e diagramas desenhados no quadro branco até wireframes e storyboards afixados na parede e que permitem eficiência quando, por exemplo, se deseja ou se precisa alterá-los, porque são "leves" o bastante para tanto.

Tais artefatos na abordagem Lean UX também são o ponto de partida para a construção de minimum viable products (MVP), um dos conceitos mais importantes na abordagem Lean UX (GOTHELF; SEIDEN, 2013, p. 55). O MVP é "a menor coisa que é possível [criar] para determinar a validade de cada uma das 
hipóteses declaradas [anteriormente]" (GOTHELF; SEIDEN, 2013, p. 56). Cria-se um MVP com o mínimo de esforço para que, expondo-o aos usuários, seja possível aprender com o público-alvo aquilo que é necessário aprender antes de investir tempo e recursos em uma ideia não comprovada. O MVP vai desde protótipos e versões reduzidas do próprio produto, até mesmo, por exemplo, uma landing page capaz de capturar o interesse do público-alvo por um produto antes mesmo de iniciar o seu desenvolvimento.

O ciclo Lean UX, apresentado na Figura 2 a seguir (à esquerda), é organizado para otimizar o aprendizado da equipe e garantir que hipóteses sejam validadas, aperfeiçoadas ou descartadas. Mas como esse ciclo é de fato praticado em um ambiente de desenvolvimento Ágil? A Figura 2 (à direita) ilustra como a abordagem Lean UX traz para dentro e ao redor do sprint atividades UX praticadas não só e isoladamente pelo UX designer, mas colaborativamente com desenvolvedores, gestores, marketing, e todos os perfis profissionais que possam contribuir para a projeção da experiência do usuário com o produto.

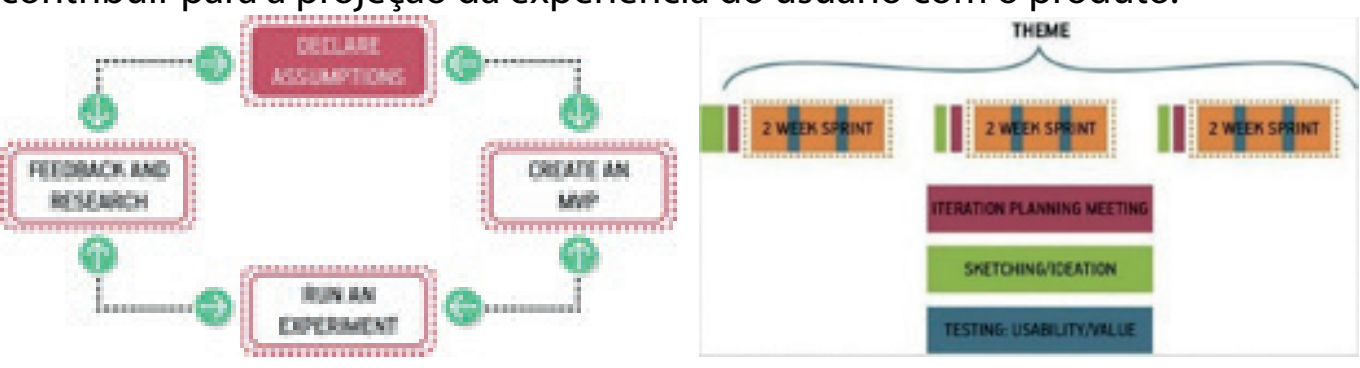

Figura 2: Ciclo Lean UX (à esquerda) e como Lean UX traz atividades UX para dentro e ao redor do sprint (à direita).

Fonte: Gothelf e Seiden, 2013, p. 18 e 100. Na Figura 2, à direita (GOTHELF; SEIDEN, 2013, p. 98-100):

- Theme: grupo de $n$ sprints relacionados entre si;

- Sketching/ Ideation: série de exercícios em equipe para declarar hipóteses que consigam resolver o problema de negócio por meio da criação de diagramas, wireframes, protótipos, etc. Parte-se do backlog de user stories e do aprendizado durante os sprints anteriores. Atividades de pesquisa também podem compor esta etapa, muito embora a colaboração dentro da equipe seja o foco;

- Iteration Planning Meeting (IPM): o resultado da etapa de sketching é o ponto de partida desta reunião. Todos os artefatos criados servem para escrever e/ou detalhar user stories a serem trabalhadas no próximo sprint;

- Testing: é o momento em que MVPs, iniciados na fase de sketching e refinados durante os sprints, entram em ação quando colocados na frente dos usuários para validar hipóteses e ideias. 


\section{INTEGRAÇÃO DO LEAN UX COM OS PLANOS DE GARRETT}

A contribuição de Garrett (2011) integrada às abordagens Lean UX e Ágil é ilustrada na Figura 3, na qual importantes processos - como sketching e testing -, momentos - como IPM, sprint - e artefatos - como user story, MVP - da abordagem Lean UX foram relacionados aos planos de Garrett (2011) - estratégia, escopo, estrutura, esqueleto e superfície.

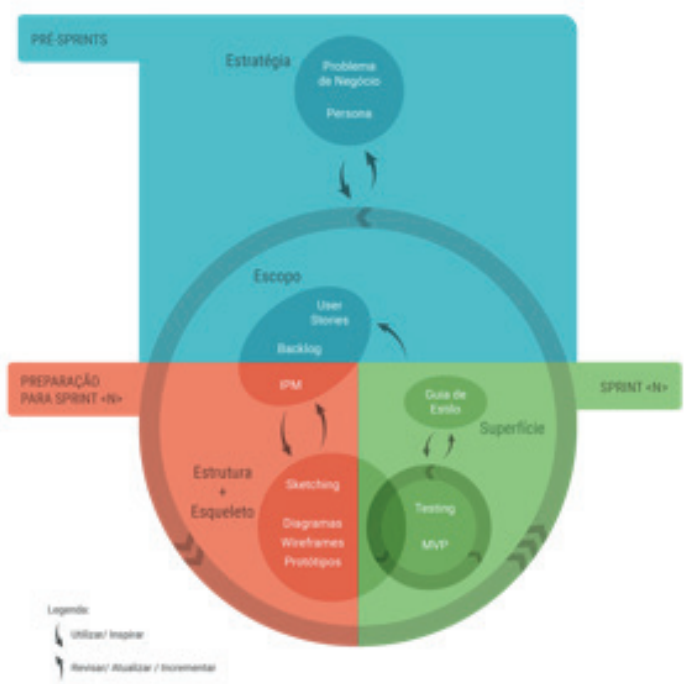

Figura 3: Integração dos planos de Garrett (2011) com as abordagens Lean UX e Ágil.

Fonte: Acervo das autoras (2017)

Numa visão ampla, a integração do framework de Garrett com as abordagens Lean UX e Ágil requer que os planos de Garrett mantenham-se abertos, sofrendo constantes atualizações e revisões no curso do projeto de um produto. Assim, adotar os princípios Lean UX e Ágil significa revisitar estes planos quando se expande o alcance do produto, quando se adiciona valor a ele, mas especialmente quando novos conhecimentos sobre o público-alvo e seus comportamentos surgem no momento de testing. O propósito das setas verticais na Figura 3 é justamente transmitir esta noção.

Antes mesmo da execução de qualquer sprint, é necessário construir um panorama sobre o problema de negócio que se pretende resolver: quais são as necessidades e comportamentos do público-alvo, como o mercado está estruturado, quais são os concorrentes e a participação de cada um, quais são os produtos similares que pretendem resolver o mesmo problema e como pretendem isso; enfim, é necessário definir os planos de estratégia e escopo do projeto, nos termos de Garrett (2011). É o que a parte superior da Figura 3 procura transmitir. Para tanto, são empregados métodos e dinâmicas de pesquisa em design mais 
tradicionais, como entrevista semi-estruturada, grupo focal, questionários, análise de estatísticas de uso de produtos (data analytics), prospecção e análise de similares, brainstorm, etc.

Mas, diferentemente de abordagens e metodologias mais tradicionais, nas abordagens Lean UX e Ágil, não se pretende na etapa "pré-sprints" esgotar todas as fontes e todas as possibilidades para entender minuciosamente cada aspecto do problema de negócio. Assim como ocorre com qualquer alternativa de design criada dentro destas abordagens, também os planos de estratégia e escopo estão sujeitos à validação, à revisão e à readequação constantes, no intuito de corrigir o curso ao longo do projeto para resolver o problema de negócio "correto" com a solução de design "mais adequada". Isto é possível devido aos inúmeros contatos com o público-alvo no projeto, especialmente no momento testing.

O combustível inicial da etapa de "preparação para o sprint", é o backlog de user stories e os resultados dos sprints anteriores, especialmente o feedback de usuários durante o momento testing, ao serem apresentados a protótipos e outros artefatos, durante testes de usabilidade e outros métodos e instrumentos de validação. Neste momento, é comum que usuários expressem interesse, entusiasmo ou mesmo sugiram determinados conteúdos, funcionalidades ou mesmo experiências. Muitas vezes, estas sugestões já estão contempladas em user stories no backlog; neste caso reavalia-se a prioridade destas user stories e a necessidade de cumpri-las já no próximo sprint. Quando estas sugestões não estão contempladas, é avaliada a necessidade de criação e inclusão de user stories no backlog que contemplem estas sugestões. No caso especial de ser o primeiro sprint, obviamente não há resultados de sprints anteriores, assim o backlog de user stories (plano de escopo) e os resultados do plano de estratégia orientam a "preparação para o sprint" e o próprio "sprint".

O objetivo principal da etapa "preparação para o sprint" é compreender o problema embutido em cada user story prevista para ser cumprida no próximo sprint e estudar alternativas para solucioná-lo. Então, por meio de esboços, wireframes e diagramas, além de outros artefatos produzidos no momento de sketching, os requisitos de cada user story são detalhados e refinados, guiando-se sempre pelas pesquisas realizadas durante o plano de estratégia e que resultaram, em última instância, na criação de personas, na escrita de uma coleção de user stories e na previsão inicial do seu escopo, nada mais do que hipóteses a serem validadas no momento testing do "sprint". Outros resultados do momento de sketching na "preparação para o sprint": 
- Revisão da prioridade de user stories, a medida em que se percebe que a resolução de uma user story entregará mais valor do que previsto inicialmente;

- Criação de novas user stories não previstas inicialmente, mas que se mostram necessárias normalmente para simplificar outra user story também do próximo sprint ou que dão conta de problemas não previstos, que estão relacionados com uma ou mais user stories a serem trabalhadas no próximos sprint.

Retomando o objetivo principal da etapa de "preparação para o sprint", na qual está inserida a etapa de sketching (ver Figura 3), em termos práticos: definir quais as user stories serão cumpridas no sprint e quais os requisitos para estas serem consideradas cumpridas (escopo). O ponto final da etapa "preparação para sprint" é o momento IPM, onde são determinadas as user stories a serem cumpridas no próximo "sprint", juntamente com seus requisitos.

$\mathrm{Na}$ Figura 3, nota-se que o momento sketching também acontece na etapa "sprint", mas seu objetivo agora é outro, é auxiliar na criação do MVP, utilizando e aproveitando os artefatos produzidos na "preparação para sprint". É também na etapa "sprint" que se constrói pouco a pouco o guia de estilos do projeto, que contém os elementos de interface reutilizáveis, paleta de cores, tipografia e qualquer outro aspecto relacionado com a aparência do produto; é a mesma preocupação do plano de superfície do Garrett (2011).

\section{APLICAÇÃO DA ABORDAGEM INTEGRADA}

A abordagem integrada, resumida na Figura 3, foi aplicada no projeto da interface de um aplicativo para plataforma Android, executado entre 2016 e 2017. O objetivo estabelecido para este projeto foi desenvolver a interface gráfica de um aplicativo para apoiar o acesso aos materiais bibliográficos pertencentes ao acervo da Biblioteca Universitária (BU) da UFSC. A seguir, um resumo das etapas realizadas neste projeto.

\subsection{ETAPA PRÉ-SPRINTS}

Neste caso aplicado, as seguintes pesquisas contribuíram para delimitar a estratégia e o escopo do projeto:

- Entrevistas semi-estruturadas com dois funcionários da BU-UFSC;

- Entrevistas semi-estruturadas com três estudantes de graduação e dois de pós-graduação da UFSC, de diferentes campos de estudo;

- Prospecção e análise de produtos similares;

- Pesquisa bibliográfica e documental em relatórios e sites.

A análise dos resultados destas pesquisas resultaram em dois artefatos prin- 
cipais utilizados e acessados ao longo de todo o projeto: duas personas, cada uma representando o estudante de graduação e o de pós, e o backlog de user stories com previsão, ainda que com status de hipóteses, de requisitos para cada uma delas. Apresenta-se na Figura 4 o backlog ao final da etapa pré-sprints, na qual destacam-se as user stories com prioridade média e alta para a

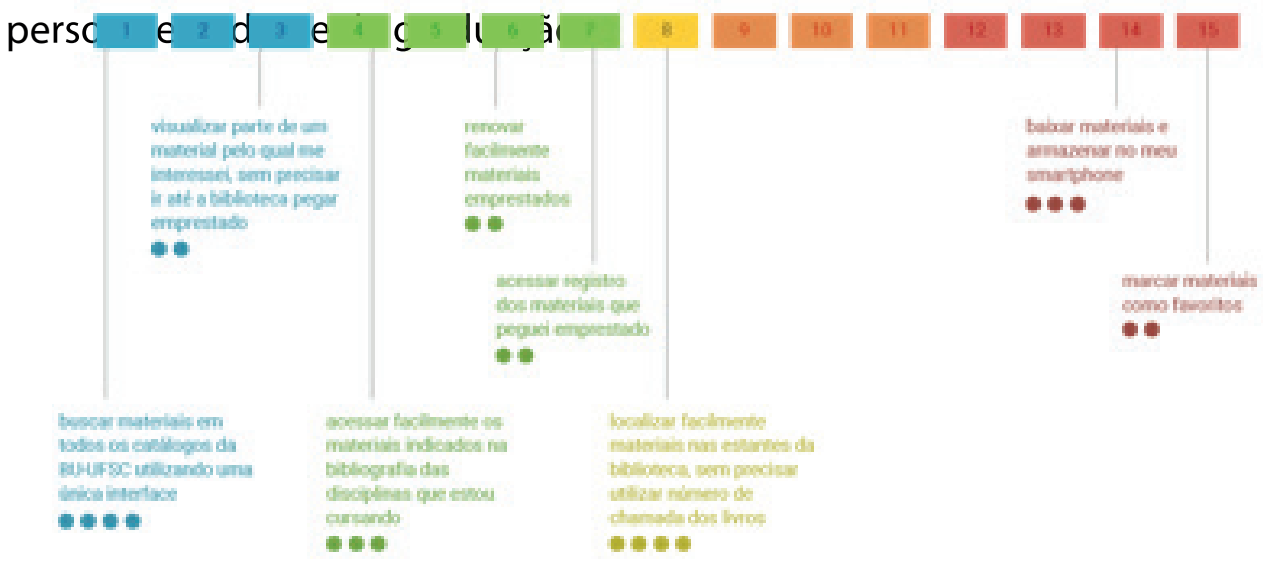

Figura 4: Representação visual do backlog de user stories ao final da etapa pré-sprints. As cores representam sprints diferentes e os círculos, os requisitos previstos para cada user story. Fonte: Acervo das autoras (2017)

Para exemplificar o formato de escrita das user stories, cita-se a user story 1: Como estudante de graduação, eu quero buscar materiais em todos os catálogos da BU-UFSC (proprietário, adquirido ou assinado) utilizando uma única interface, porque eu perco menos tempo, já que precisarei realizar determinada busca uma única vez.

\subsection{ETAPA PREPARAÇÃO PARA SPRINT}

A preparação para sprint teve duração de um par de dias antes de cada um dos dois sprint e envolveu especialmente esboçar em papel e lápis alternativas de design para cada user story definida para cada um dos dois sprints. A Figura 5, à esquerda, traz um destes esboços. Os esboços serviram não só para estudar diferentes alternativas de layout, elementos de interface, conteúdos de texto, imagem e ícones, mas principalmente para detalhar e definir quais user stories seriam cumpridas no sprint.

\subsection{ETAPA SPRINT}

Protótipos funcionais de média e alta fidelidade foram desenvolvidos em HTML5 para testar as hipóteses formuladas - os requisitos das user stories de cada sprint - em cinco rodadas de testes de usabilidade, que envolveram ao 
todo a participação de doze estudantes de graduação da UFSC. Cada sprint tomou entre duas e três semanas. A Figura 5 mostra a evolução do protótipo em decorrência da incorporação do feedback de usuários coletados durante as cinco rodadas de testes de usabilidade aplicados nos dois sprints. Também mostra decisões parciais com relação ao design visual, que posteriormente foram
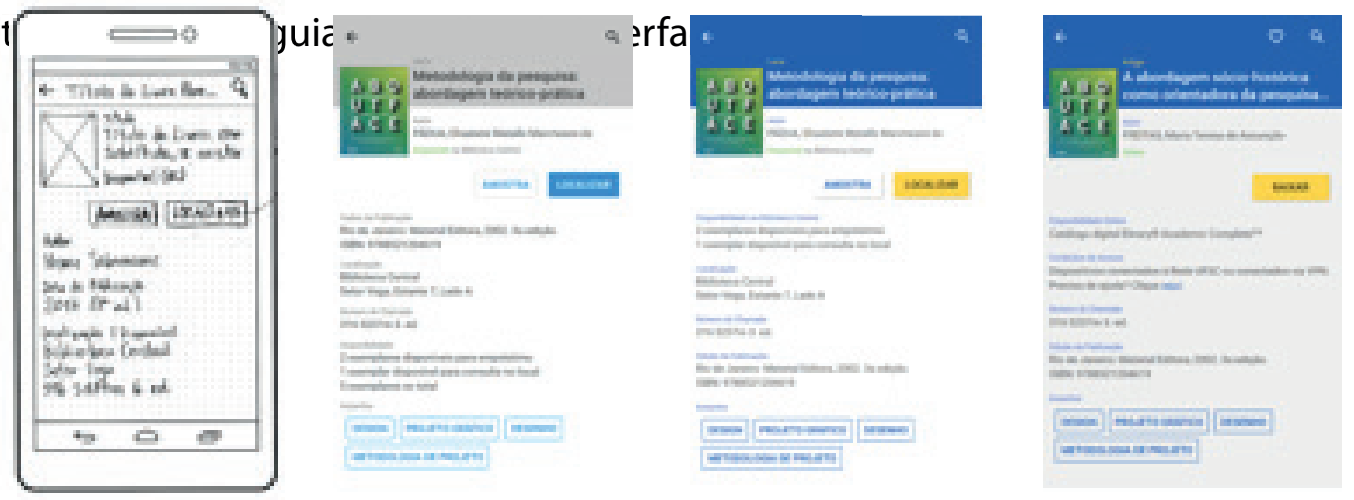

Figura 5: Evolução de uma das telas do protótipo, desde um de seus esboços (à esquerda) feito na etapa preparação para sprint. Fonte: Acervo das autoras (2017)

Apesar de serem previstos cinco sprints neste projeto, por limitação de tempo, apenas dois foram executados. Assim, ao final do projeto, um retrato mais fiel das user stories executadas é apresentado na Figura 6.

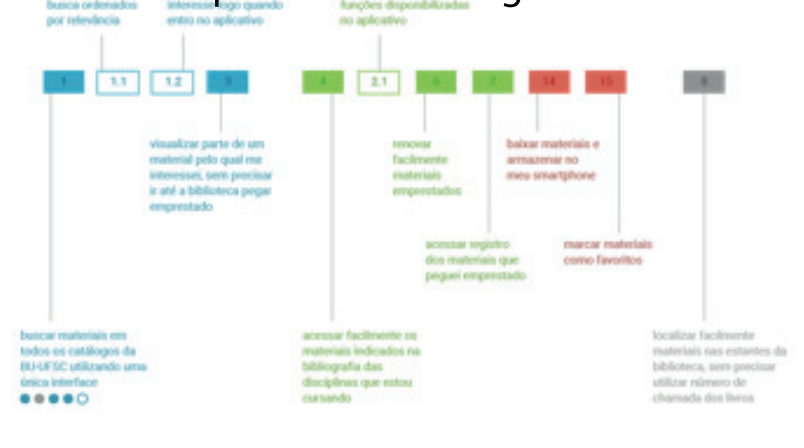

Figura 6: Representação visual de user stories executadas ao final do projeto. User stories com fundo branco foram criadas e requisitos foram revisados (círculos) na etapa preparação para sprint, em cada iteração. A user story 8, de prioridade alta, não foi executada.

Fonte: Acervo das autoras (2017)

\section{CONSIDERAÇÕES FINAIS}

A aplicação da abordagem integrada Lean UX com o framework de Garrett no projeto do aplicativo descrito anteriormente resultou em contribuições já esperadas para abordagens ágeis, mas também outras exclusivamente decorrentes da integração. 
$\mathrm{Na}$ integração das abordagens, a conexão entre os diversos momentos e artefatos da abordagem Lean UX com os planos de Garrett (2011) estimulou a elevação de consciência sobre o papel principal de cada etapa inserida no projeto como um todo.

A visibilidade das disciplinas importantes ao design de sistemas interativos, promovida pelos planos de Garrett (2011), proporcionou maior confiança na tomada de decisão nos sprints e entre sprints. Assim, por exemplo, no estudo de alternativas de layout por meio de esboços durante o momento sketching, recorreu-se aos fundamentos das disciplinas dos planos de estrutura e esqueleto: design de interação, arquitetura da informação, design de interface, design de navegação e design de informação.

Outros benefícios prometidos pelas abordagens Lean UX e Ágil também foram observados no projeto do aplicativo:

- Fluxo constante de feedback de usuários (público-alvo) durante todo o projeto; - Revisão de prioridade de user stories e incorporação de novas user stories (requisitos do projeto - plano de escopo) no curso do projeto, fundamentada pelo feedback de usuários em testes de usabilidade.

Como o projeto, no qual a abordagem integrada foi aplicada, não contou com a implementação e produção do aplicativo, é necessário experimentá-la em projetos nos quais especialmente o papel de desenvolvedores esteja presente, muito embora outros perfis profissionais sejam essenciais nos projetos de sistemas interativos. Desse modo, será possível verificar a utilidade desta abordagem para buscar a visibilidade das disciplinas importantes ao design destes sistemas e, assim, aumentar a confiança na prática profissional do designer de experiência do usuário.

\section{REFERÊNCIAS}

ASHMORE, Sondra; RUNYAN, Kristin. Introduction to Agile Methods. Upper Saddle River, NJ: Pearson Education, c2015.

ASSOCIAÇÃO BRASILEIRA DE NORMAS TÉCNICAS. NBR ISO 9241 - Parte 210: Ergonomia da interação humano-sistema: Projeto centrado no ser humano para sistemas interativos. Rio de Janeiro, 2011. 38p.

GARRETT, Jesse James. Elements of user experience, the: user-centered design for the web and beyond. Pearson Education, 2011. 2a edição. 
GOTHELF, Jeff; SEIDEN, Josh. Lean UX: Applying lean principles to improve user experience. “O’Reilly Media, Inc.”, 2013.

NIELSEN, Jakob. Iterative User Interface Design. IEEE Computer, v. 26, n. 11, p. 32-41, novembro, 1993. Disponível em: <https://www.nngroup.com/articles/ iterative-design/>. Acesso em: 20 maio 2017.

VERSIONONE. 10th Annual State of Agile Report, the. 2016. Disponível em: $<$ https://explore.versionone.com/state-of-agile/versionone-10th-annual-state-of-agile-report-2>. Acesso em: 20 junho 2017.

Berenice Santos Gonçalves é Doutora em Engenharia de Produção pela UFSC (2004) e Mestre em Artes Visuais pela UFRGS (1997). Atua como professor Associado na UFSC onde ministra disciplinas nas áreas de Ergonomia e usabilidade e Design Digital para o curso de Graduação em Design. Atua no Programa de Pós-Graduação em Design (Mestrado e Doutorado). Desenvolve pesquisas nas linhas de Mídia e Mídia e Tecnologia a partir dos seguintes eixos: "Interação e Interface", "Design Editorial no contexto das tecnologias digitais" e "Hipermídia para aprendizagem". Lidera e participa de grupos de pesquisa referendados pelo CNPq. Tem publicações nas principais revistas científicas nacionais da área de Design: Infodesign, Estudos em Design, Educação Gráfica, Design e Tecnologia, Ergodesign, entre outras.

Andréa Bonette Ferrari é Bacharel em Design pela UFSC (2017) e Licenciada em Física pela USP (2009). Vem atuando desde 2010 em projetos de websites e aplicativos para educação à distância, inicialmente com foco em UI Design e atualmente com foco em UX Design. Estudou e estagiou nos EUA pelo Programa Ciência Sem Fronteiras entre 2015 e 2016, quando teve oportunidade de atuar como UX Designer em startup de tecnologia, principalmente planejando e aplicando testes de usabilidade em ambiente de desenvolvimento Agile. Outras informações profissionais podem ser encontradas no Linkedln. 